\title{
Probióticos en enfermedad celíaca: ¿estamos listos para su aplicación en la práctica clínica?
}

\author{
Gastón H Rueda [ • M Inés Pinto-Sánchez \\ Farncombe Family Digestive Health Research Institute, McMaster University, Ontario, Canada.
}

Acta Gastroenterol Latinoam 2021;51(4):394-402

Recibido: 21/09/2021 / Aceptado: 28/10/2021 / Publicado online: 13/12/2021 / https://doi.org/10.52787/GQME9827

\section{Resumen}

Los probióticos, microorganismos vivos que producen un efecto beneficioso en la salud, son un posible complemento de la dieta libre de gluten en el tratamiento de la enfermedad celíaca. En diversos estudios clínicos se demostró que pacientes celiacos tratados con probióticos mejoraron sus sintomas gastrointestinales. Aunque los mecanismos de acción de los probióticos en la enfermedad celiaca son poco claros, investigaciones realizadas en roedores proponen diferentes mecanismos, tales como la modulación de la microbiota intestinal y el sistema inmune, o a través de la producción de proteasas. Realizamos una revisión de la literatura para abordar la evidencia actual sobre la eficacia de los probióticos en el tratamiento de la enfermedad celiaca, posibles mecanismos de acción y áreas de interés para futuros estudios de investigación.

Palabras claves. Probióticos, enfermedad celíaca.

Correspondencia: Gastón Horacio Rueda

1280 Main Street West, Hamilton, ON L8S 4K1, Health Sciences Centre, 3N51 - E. Farncombe Family Digestive Health Research Institute, McMaster University.

Correo electrónico: ruedag@mcmaster.ca

\section{Probiotics in Celiac Disease: Are We Ready for Their Use in Clinical Practice?}

\section{Summary}

Probiotics, live microorganisms that produce a beneficial effect on health, are a possible supplement to a gluten-free diet in the treatment of celiac disease. Several clinical studies have shown that celiac patients treated with probiotics improved their gastrointestinal symptoms. Although the mechanisms of probiotics in celiac disease are unclear, preclinical studies in mice suggest different mechanisms, such as the modulation of the intestinal microbiota and the immune system, or through the production of proteases. We conducted a review of the literature to address the current evidence on the efficacy of probiotics in the treatment of celiac disease, possible mechanisms of action, and areas of interest for future research studies.

Keywords. Probiotics, celiac disease.

\footnotetext{
Abreviaturas

EC: Enfermedad celíaca.

CeD: Celiac disease.

DLG: Dieta libre de gluten.

GSRS: Gastrointestinal Symptom Rating Scale.

CSI: Celiac Symptom Index.

IBS-SSS: Irritable Bowel Syndrome Severity Scoring System.

TNF: Factor de necrosis tumoral.
} 
AhR: Receptor de hidrocarburos de arilo.

ATI: Inhibidor de amilasa tripsina.

IgA tTG: Anticuerpos antitransglutaminasa IgA.

IgA DGP: Antipéptido deamidado de gliadina IgA.

\section{Introducción}

Los probióticos son microorganismos vivos que una vez administrados en cantidad adecuada, provocan un beneficio para la salud de su huésped. ${ }^{1}$ La mayoría de los probióticos provienen de la microbiota intestinal normal de un individuo sano. ${ }^{2}$ Los probióticos han generado un gran impacto en la función de barrera intestinal en diferentes patologías gastrointestinales, desde desórdenes funcionales hasta enfermedades inflamatorias inmunomediadas. Actúan interactuando con el huésped y modulando el sistema neuroendocrino e inmune, así como también mediante la digestión de antígenos dietarios, que influye directamente en la tolerancia a alimentos. ${ }^{3}$

La enfermedad celíaca (EC) es una enfermedad sistémica inmunomediada que ocurre en individuos genéticamente predispuestos, y se caracteriza por presentar una enteropatía inflamatoria crónica producida por la ingesta de gluten. ${ }^{4}$ Es uno de los trastornos digestivos más comunes en todo el mundo y afecta alrededor del $1 \%$ de la población mundial. ${ }^{5}$ En Latinoamérica la prevalencia estimada es similar a la de Europa, debido probablemente a que ambas poblaciones comparten la susceptibilidad genética de portar los haplotipos HLA DQ2 y/o DQ8. ${ }^{6}$ El diagnóstico y su tasa de prevalencia se han incrementado drásticamente, y esto no se explica solamente por un aumento en la tasa de detección, ${ }^{7}$ lo cual sugiere que en el desarrollo de la EC están involucrados otros factores ambientales. El manejo de la enfermedad, tanto al momento del diagnóstico como el seguimiento durante años luego de su detección, genera importantes costos económicos para el sistema de salud. ${ }^{8}$

El único tratamiento aceptado para la EC es la dieta libre de gluten (DLG), ${ }^{9}$ que no es lo suficientemente efectiva para controlar los síntomas a largo plazo. ${ }^{10}$ Se estima que más de un $40 \%$ de los pacientes con EC tendrán síntomas persistentes a pesar de adherir a la DLG. ${ }^{11-12} \mathrm{Si}$ bien existen investigaciones que evalúan nuevas terapéuticas adyuvantes a la DLG, ${ }^{13}$ el manejo clínico actual de la EC en pacientes que persisten con síntomas es un desafío.

La microbiota intestinal tiene un rol importante en la digestión, tanto mediante la síntesis de nutrientes y metabolitos como en la modulación del sistema inmune y el mantenimiento de la integridad de la mucosa intestinal. ${ }^{14}$ Se han asociado cambios en la composición de la función de la microbiota intestinal con enfermedades inflamatorias crónicas, entre ellas la EC. ${ }^{15}$ Más aún, la persistencia de síntomas en pacientes con EC tratados con DLG ha sido relacionada con cambios en la composición de la microbiota intestinal, ${ }^{16}$ lo cual abre la posibilidad de nuevas terapéuticas en EC. Estudios con probióticos en poblaciones pediátricas y adultas con EC mostraron mejoría de síntomas a partir de diferentes mecanismos, que incluyeron efectos inmunomoduladores, cambios en la permeabilidad intestinal y modificaciones en la inhibición del crecimiento de bacterias patógenas. ${ }^{17-19}$ A continuación, evaluamos la evidencia actual sobre el efecto de los probióticos en diferentes aspectos relacionados a la EC.

\section{Efecto de los probióticos en la mejoría de sín- tomas de pacientes con EC}

En una revisión sistemática con metaanálisis publicada recientemente, ${ }^{20}$ se evaluó la evidencia basada en estudios clínicos randomizados controlados con probióticos y sus efectos en diferentes parámetros, incluyendo síntomas gastrointestinales y mejoría de la calidad de vida. La evidencia sobre el efecto de los probióticos en los síntomas gastrointestinales proviene de cuatro estudios clínicos randomizados realizados en adultos con EC. Francavilla y col. ${ }^{21}$ demostraron una disminución de la gravedad de los síntomas gastrointestinales de tipo síndrome de intestino irritable en pacientes con EC que seguían una DLG estricta y fueron tratados con Lactobacillus y Bifidobacteria durante seis semanas. Sin embargo, este estudio ${ }^{21}$ no demostró cambios en la calidad de vida de los pacientes tratados con probióticos en comparación con el grupo placebo.

Por otra parte, esos resultados difieren de los hallazgos de otro estudio realizado por Harnett y col. ${ }^{22}$ en el que se administró la mezcla de probióticos "VSL\#3" (Streptococcus thermophilus, Bifidobacterium breve, Bifidobacterium longum, Bifidobacterium infantis, Lactobacillus acidophilus, Lactobacillus plantarum, Lactobacillus paracasei y Lactobacillus delbrueckii subsp. bulgaricus) por un período de 12 semanas. En esta investigación no se encontró mejoría de los síntomas gastrointestinales específicos de EC ni en la calidad de vida de la población estudiada.

Smecuol y col. ${ }^{23}, 24$ evaluaron el efecto del probiótico Bifibacterium infantis NLS super strain en: a) pacientes con diagnóstico reciente de $\mathrm{EC},{ }^{23} \mathrm{y}$ b) pacientes con $\mathrm{EC}$ tratados con DLG que presentaban síntomas persistentes. ${ }^{24}$ Ambos grupos demostraron mejoría en los síntomas gastrointestinales utilizando la escala GSRS (Gastrointestinal Symptom Rating Scale), ${ }^{23}$ así como en los síntomas específicos de EC evaluados con la escala CSI (Celiac Symptom Index). ${ }^{24} \mathrm{El}$ análisis combinado de dos estudios randomizados controlados ${ }^{21,} 23$ demostró una disminu- 
ción en los síntomas gastrointestinales utilizando la escala GSRS (MD $=-28,74 \%, 95 \%$ CI $-43,96 \%$ a $-13,52 \%$, $p=0,0002)$ en pacientes tratados con probióticos comparados con placebo. Sin embargo, investigaciones que utilizaron otros cuestionarios validados en síntomas específicos de EC (CSI) o de síndrome de intestino irritable (Irritable Bowel Syndrome Severity Scoring System - IBS-SSS), no demostraron cambios significativos luego del tratamiento con probióticos. ${ }^{22-24}$

\section{Efecto de los probióticos en la inducción de cambios de la microbiota intestinal en EC}

Existen numerosos eventos tempranos en la vida que pueden provocar la alteración de la microbiota intestinal en la EC. Los mismos incluyen: predisposición genética, tiempo y la cantidad de gluten introducido en la dieta, tipo de parto, uso de antibióticos, así como también el desarrollo de infecciones en la infancia. ${ }^{25-29}$

En estudios realizados en la materia fecal de niños con predisposición genética HLA-DQ2, se encontró un aumento de Firmicutes, Proteobacteria y disminución de Bifidobacteria, sugiriendo que el genotipo podría modular y determinar la composición de la microbiota intestinal en esa población, contribuyendo así al incremento del riesgo a desarrollar EC. ${ }^{30}$ Por otra parte, en niños con EC activa se encontraron cambios en la microbiota intestinal, con una reducción del género Lactobacillus, que se restauró parcialmente luego de la dieta libre de gluten. ${ }^{31}$

Los cambios en la microbiota intestinal también demostraron tener un papel importante en los diferentes estadios y manifestaciones clínicas de la EC, mostrando mayor abundancia de Proteobacteria en pacientes sintomáticos con $\mathrm{EC},{ }^{32}$ sugiriendo que la disbiosis intestinal en esta población podría ser responsable de los síntomas persistentes. Si bien los estudios expuestos describen diferencias en la microbiota intestinal en pacientes con EC, la relación causa y efecto no está clara.

Entender los perfiles de la microbiota intestinal en el contexto de EC ha sido importante para estudiar los mecanismos que podrían ser regulados por ciertos probióticos. Un estudio clínico randomizado de 49 niños con EC tratados con DLG evaluó la eficacia de dos cepas de Bifidobacterium breve (B632 y BR03) en el restablecimiento de microbiota intestinal, demostrando que el suplemento probiótico generaba un incremento en Actinobacteria, así como también la restauración de la relación Firmicutes/ Bacteroidetes. $^{33}$

Por el contrario, otro estudio randomizado de $45 \mathrm{pa}$ cientes con EC tratados con DLG y probiótico VSL\#3, no demostró diferencias significativas en el recuento de la microbiota fecal o cambios en la severidad de los síntomas luego de dos semanas de tratamiento. ${ }^{22}$

En el citado metaanálisis de reciente publicación ${ }^{20}$ se evaluaron los cambios de la microbiota fecal, cuantificando la abundancia absoluta en los géneros Bifidobacteria y Lactobacillus en la totalidad de estos estudios clínicos. ${ }^{21,} 22,33,34$ Se demostró un incremento significativo en las especies de Bifidobacteria en el grupo tratado con probióticos, comparado con placebo (MD $=0,85,95 \%$ CI $\left.0,38-1,32, \mathrm{I}^{2}=62 \%, p=0,0003\right)$, no así en las cepas de Lactobacillus. ${ }^{20}$ Los niveles de Bifidobacteria fueron detectables en muestras fecales incluso luego de seis semanas de discontinuación del probiótico utilizado. ${ }^{21}$

\section{Efecto de los probióticos en la modulación de la respuesta inmune $y$ sobre los marcadores inflamatorios en pacientes con EC}

La microbiota intestinal ha demostrado regular aspectos claves en la inmunidad innata y adaptativa, así como también en la reducción de los procesos inflamatorios inducidos por gluten y mediados por diferentes mecanismos. ${ }^{35}$ Ciertas bacterias han demostrado tener la capacidad de hidrolizar péptidos derivados del gluten, reduciendo claramente su inmunogenicidad. ${ }^{36}$ Bacterias patógenas como Pseudomona aeruginosa, presente en pacientes con EC, han demostrado ser capaces de potenciar la inmunogenicidad de los péptidos 33-mer, uno de los péptidos responsables de desencadenar la respuesta inmune típica de EC; mientras que especies de Lactobacillus aislados de sujetos controles revirtieron esta reacción, resultando en la disminución en la activación de linfocitos T. ${ }^{37}$ Estudios preclínicos en modelos de ratones demostraron efectos positivos de Bifidobacterium longum CECT 7347 y del probiótico Bifidobacterium breve, disminuyendo la producción de citoquinas proinflamatorias y linfocitos T CD $4+.^{38}$

Por otra parte, TNF-alfa ha resultado ser también un mediador importante en la activación del sistema inmune, produciendo un daño epitelial intestinal en pacientes con EC. La disminución en la producción de esta citoquina inducida por los probióticos, junto a una DLG, podría resultar en un efecto beneficioso en el manejo de la EC. ${ }^{39}$

En 2018, un estudio clínico randomizado doble ciego, demostró que la mezcla de las cepas del probiótico Bifidobacterium breve (DSM 16604 y DSM 24706) fue capaz de modular la producción de ácido acético y de ácidos grasos de cadena corta luego de tres meses de administración, comparado con placebo, en niños con EC. Se observó que tuvieron un rol importante no solo en la restauración de la microbiota intestinal, sino también en 
la modulación de TNF-alfa. ${ }^{40}$ Sin embargo, el efecto de los probióticos en niveles séricos de TNF-alfa no ha sido confirmado en otros estudios clínicos. ${ }^{17,}$ 20, 34 Finalmente, un estudio reciente ${ }^{48}$ demostró que los pacientes celíacos presentaban alteraciones en el metabolismo del Receptor de hidrocarburos de arilo (AhR), y la administración de probiótico Lactobacillus reuteri, productor de ligandos para el receptor AhR a partir del aminoacido esencial triptofano, redujo la inflamación en ratones expuestos al gluten, lo cual abre nuevas posibilidades en el tratamiento de EC.

\section{Efecto de los probióticos en la barrera intestinal}

Diferentes estudios preclínicos evaluaron los efectos directos e indirectos de los probióticos en la integridad de la mucosa intestinal y en la mejoría de la permeabilidad intestinal previamente alterada por la ingesta de gluten.

Algunos probióticos, como Lactobacillus fermentum y Bifidobacterium lactis, demostraron reducir el daño ocasionado por los péptidos derivados del gluten en estudios in vitro (células Caco-2).${ }^{41}$ Un estudio similar in vitro demostró que el incremento significativo del transporte paracelular de lactulosa inducido por la gliadina fue revertido luego de la administración de Lactobacillus rhamnosus $G G .^{42}$ Por otra parte, estudios en ratones sensibilizados con gliadina demostraron que la administración de Bifidobacterium longum CECT $7347^{38}$ y Saccharomyces boulardii $K K 1,{ }^{43}$ fueron capaces de disminuir la enteropatía inmunomediada típica en EC. Interesantemente, estudios en modelos animales genéticamente susceptibles a desarrollar EC demostraron un rol importante en la detección de bacterias productoras de proteasas: Lactobacillus demostró tener función degradadora de los inhibidores de amilasa tripsina (ATI); ${ }^{44}$ y Bifidobacterium longum NCC2705 demostró ser productor de inhibidores de serina proteasas (serpinas), los cuales podrían tener un papel en la prevención de la enteropatía inmunomediada por gluten. ${ }^{18}$

En cuanto a estudios clínicos, solo uno de ellos investigó la función de permeabilidad intestinal en pacientes sintomáticamente activos luego de la administración de probiótico Bifidobacteria NLS super strain, evaluado por método indirecto de lactulosa-manitol, el cual no demostró cambios en la permeabilidad intestinal antes y después del probiótico. ${ }^{23}$ Sin embargo, es posible que la baja especificidad y sensibilidad de esta prueba y el tamaño de la muestra hayan influido en los resultados. Si bien las investigaciones presentadas sugieren que la administración de probióticos modificó la función epitelial y la permeabilidad intestinal, en ninguno de los estudios clínicos disponibles se realizó biopsia duodenal antes y después de la suplementación con probioticos. Por lo tanto, se desconoce si los probioticos indujeron cambios en la mucosa intestinal en pacientes con EC.

\section{Efecto de los probióticos en la serología espe- cífica de la EC}

Dos estudios clínicos randomizados controlados evaluaron el efecto de la suplementación de Bifidobacterium infantis NLS super strain, ${ }^{23}$ y de Lactiplantibacillus plantarum HEAL9 Lacticaseibacillus paracase $i^{45}$ en los niveles de anticuerpos antitransglutaminasa IgA (IgA tTG) y anti-peptidos deamidados de gliadina IgA (IgA DGP). Los resultados de estos estudios no observaron cambios significativos en los niveles serológicos de estos anticuerpos antes y después del tratamiento comparado con placebo.

\section{Perfil de seguridad de los probióticos en EC}

Varios estudios clínicos evaluaron posibles efectos adversos relacionados a los probióticos. ${ }^{17,}$ 21, 22, 24 Ninguno de los estudios encontró diferencias significativas en el número de eventos adversos bajo suplemento con probióticos, comparado con placebo; se considera por lo tanto que los probióticos estudiados son seguros en la EC.

\section{¿Hay alguna cepa de los probióticos que sea más beneficiosa en la EC?}

Si bien estudios clínicos en población adulta y niños sugieren un efecto beneficioso con el uso de ciertos probióticos, como son Bifidobacterium y Lactobacillus, ${ }^{17}, 21,22,24,33,40,45$ el efecto de éstos son limitados a poblaciones específicas y el mecanismo de acción no está claro.

Estudios recientes demostraron que se pueden encontrar bacterias que degradan el gluten en el intestino delgado de humanos. ${ }^{46}$ Entre todos los posibles géneros bacterianos presentes en la microbiota intestinal, Lactobacilli y Bifidobacterium spp. han demostrado compartir la actividad proteolítica y peptidolítica más extensa involucrada en la descomposición del gluten, con lo cual podrían ser de posible uso como suplemento probiótico en pacientes con EC. ${ }^{47}$

Sin embargo, el grado de certeza y la calidad de la evidencia científica actual sobre el efecto de los probióticos en diferentes parámetros de la EC es bajo (Figura 1), y debido a la heterogeneidad en el desarrollo de los estudios, no es posible definir aún cepas específicas que puedan ser más beneficiosas que otras para el tratamiento de pacientes con EC. 
Figura 1. Certeza de las recomendaciones sobre el efecto de los probióticos en la enfermedad celiaca de acuerdo a la calidad de la evidencia cientifica utilizando la escala de evaluación GRADE.

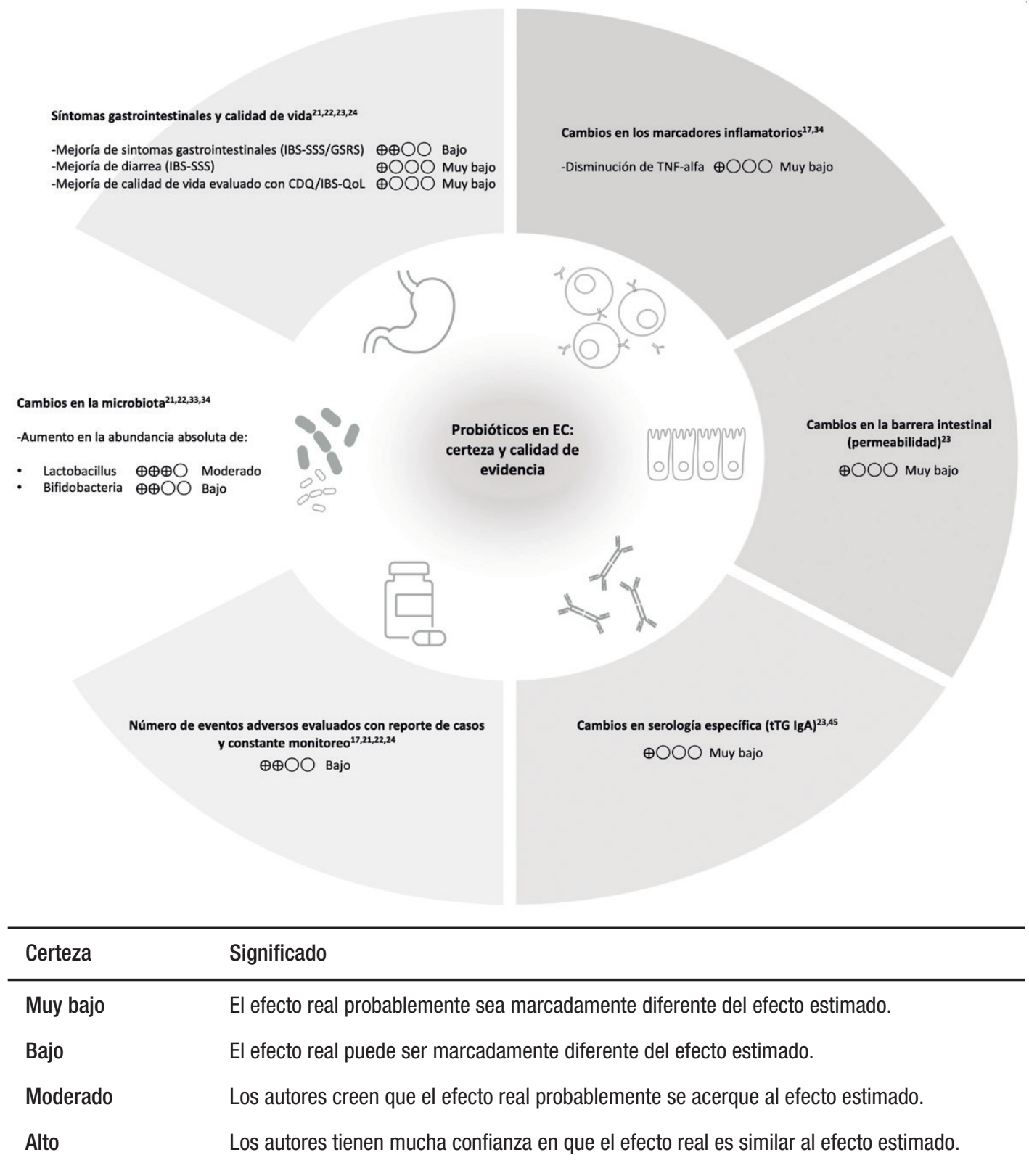

\section{Áreas desconocidas, brechas en la investiga- ción relacionada con probióticos en EC. Guía para futuros estudios}

Si bien existen estudios en población adulta y pediátrica sobre el efecto de probióticos en la EC como tratamiento adyuvante a la DLG (Tabla 1), la evidencia científica en el efecto de los probióticos en diferentes aspectos de la EC tiene un bajo grado de certeza (Figura 1). Se necesitan estudios a mayor escala para poder confirmar el efecto beneficioso del uso de probióticos en la EC, así como los mecanismos por los cuales inducen mejoría sin- tomática. Además, es poco claro qué género y especie de probióticos, dosis ideal e intervalo de tratamiento se deberían proponer en la población celíaca. Aunque existen estudios en ratones que demostraron resultados promisorios por el efecto beneficioso de bacterias productoras de proteasas ${ }^{46,47}$ o por la modificación de la activación de los receptores de hidrocarburos de arilo, ${ }^{48}$ se necesitan estudios clínicos que evalúen el uso de estos probióticos en pacientes celíacos, particularmente en el subgrupo de pacientes con síntomas persistentes y aquellos expuestos a mínimas cantidades de gluten. 
Tabla 1. Características de los ensayos clínicos randomizados

\begin{tabular}{|c|c|c|c|c|}
\hline Autor (ref) & $\begin{array}{l}\text { País de origen/ } \\
\text { número de centros }\end{array}$ & Población & Intervención & Parámetros evaluados \\
\hline $\begin{array}{l}\text { Francavilla y } \\
\text { col. }^{21}\end{array}$ & $\begin{array}{l}\text { Italia } \\
\text { Un solo centro }\end{array}$ & $\begin{array}{l}109 \text { adultos con } \\
\text { diagnóstico de EC } \\
\text { (biopsia) en DLG por } \\
\text { más de } 2 \text { años con } \\
\text { síntomas persistentes }\end{array}$ & $\begin{array}{l}\text { Mezcla de } 4 \times 10^{10} \text { UFC } \\
\text { Lactobacillus casei } \\
\text { LMG 101/37 P-17504, Lactobacillus } \\
\text { plantarum CECT } 4528 \text {, Bifidobacterium } \\
\text { animalis subsp. lactis Bi1 LMG } \\
\text { P-17502, Bifidobacterium breve Bbr8 } \\
\text { LMG P-17501, y B. breve Bl10 LMG } \\
\text { P-17500 diariamente por } \\
6 \text { semanas vs placebo (sachet) }\end{array}$ & $\begin{array}{l}\text { 1. Mejoría en síntomas Gl (GSRS, IBS- } \\
\text { SSS) } \\
\text { 2. Cambios en calidad de vida (IBS-QOL) } \\
\text { 3. Número de efectos adversos } \\
\text { 4. Cambios en la microbiota intestinal }\end{array}$ \\
\hline $\begin{array}{l}\text { Harnett y } \\
\text { col. }{ }^{22}\end{array}$ & $\begin{array}{l}\text { Australia } \\
\text { Un solo centro }\end{array}$ & $\begin{array}{l}45 \text { adultos con } \\
\text { diagnóstico de EC } \\
\text { (biopsia) en DLG por } \\
\text { más de un año con } \\
\text { síntomas persistentes }\end{array}$ & $\begin{array}{l}4,5 \times 10^{11} \text { UFC de VSL\#3, dos veces } \\
\text { al día por } 12 \text { semanas vs placebo } \\
\text { (sachet) }\end{array}$ & $\begin{array}{l}\text { 1. Mejoría en síntomas Gl (CDQ-GI) } \\
\text { 2. Cambios en calidad de vida (CDQ) } \\
\text { 3. Número de efectos adversos } \\
\text { 4. Cambios en la microbiota intestinal }\end{array}$ \\
\hline $\begin{array}{l}\text { Klemenak y } \\
{\text { col. }{ }^{17}}\end{array}$ & $\begin{array}{l}\text { Eslovenia } \\
\text { Un solo centro }\end{array}$ & $\begin{array}{l}46 \text { niños con diagnóstico } \\
\text { de EC (biopsia) en DLG } \\
\text { por } 0.5-15 \text { años }\end{array}$ & $\begin{array}{l}\text { Mezcla de } 2 \times 10^{9} \text { UFC de } \\
\text { Bifidobacterium breve BR03 y B. breve } \\
\text { B632 diariamente por } 3 \text { meses vs } \\
\text { placebo (sachet) }\end{array}$ & $\begin{array}{l}\text { 1. Cambios en TNF-a } \\
\text { 2. Cambios en IL-10 } \\
\text { 3. Número de efectos adversos } \\
\text { 4. Cambios en la microbiota intestinal }\end{array}$ \\
\hline $\begin{array}{l}\text { Olivares y } \\
\text { col. }^{34}\end{array}$ & $\begin{array}{l}\text { España } \\
\text { Un solo centro }\end{array}$ & $\begin{array}{l}33 \text { niños con diagnóstico } \\
\text { nuevo de EC (biopsia), } \\
\text { empezando DLG }\end{array}$ & $\begin{array}{l}1 \times 10^{9} \text { UFC de Bifidobacterium } \\
\text { longum CECT } \\
7347 \text { diariamente por } 3 \text { meses vs } \\
\text { placebo (capsulas) }\end{array}$ & $\begin{array}{l}\text { 1. Cambios en TNF-a } \\
\text { 2. Cambios en IL-10 } \\
\text { 3. Número de efectos adversos }\end{array}$ \\
\hline $\begin{array}{l}\text { Oscarsson y } \\
\text { col. }^{45}\end{array}$ & $\begin{array}{l}\text { Suecia } \\
\text { Un solo centro }\end{array}$ & $\begin{array}{l}78 \text { niños con diagnóstico } \\
\text { de EC (niveles elevados } \\
\text { de tTGA y riesgo } \\
\text { genético positivo) }\end{array}$ & $\begin{array}{l}\text { Mezcla de } 1 \times 10^{10} \text { UFC de } \\
\text { Lactobacillus plantarum HEAL9 y L. } \\
\text { paracasei } 8700: 2 \text { diariamente por } \\
6 \text { meses vs placebo (capsulas) }\end{array}$ & $\begin{array}{l}\text { 1. Cambios en IgA-tTG e lgG-tTG } \\
\text { 2. Cambios en la microbiota intestinal } \\
\text { 3. Identificación de correlaciones entre } \\
\text { microbiota fecal y IgA-tTG e lgG-tTG }\end{array}$ \\
\hline $\begin{array}{l}\text { Quagliariello y } \\
\text { col. }^{33}\end{array}$ & $\begin{array}{l}\text { Eslovenia } \\
\text { Un solo centro }\end{array}$ & $\begin{array}{l}40 \text { niños con diagnóstico } \\
\text { de EC (biopsia) en DLG } \\
\text { por } 0.5-15 \text { años }\end{array}$ & $\begin{array}{l}\text { Mezcla de } 2 \times 10^{9} \text { UFC de Bifidobacterium } \\
\text { breve BR03 y B. breve B632 } \\
\text { diariamente por } 3 \text { meses vs placebo } \\
\text { (sachet) }\end{array}$ & 1. Cambios en la microbiota intestinal \\
\hline $\begin{array}{l}\text { Primec y } \\
\text { col. }{ }^{40}\end{array}$ & $\begin{array}{l}\text { Eslovenia } \\
\text { Un solo centro }\end{array}$ & $\begin{array}{l}40 \text { niños con diagnóstico } \\
\text { de EC (biopsia) en DLG } \\
\text { por } 0.5-15 \text { años }\end{array}$ & $\begin{array}{l}\text { Mezcla de } 2 \times 10^{9} \text { UFC de Bifidobacterium } \\
\text { breve BR03 y B. breve B632 diariamente } \\
\text { por } 3 \text { meses vs placebo (sachet) }\end{array}$ & $\begin{array}{l}\text { 1. Correlación entre microbiota fecal, } \\
\text { TNF-a sérico y ácidos grasos de cadena } \\
\text { corta en materia fecal }\end{array}$ \\
\hline $\begin{array}{l}\text { Smecuol y } \\
\text { col. }^{23}\end{array}$ & $\begin{array}{l}\text { Argentina } \\
\text { Un solo centro } \\
\text { Estudio clínico } \\
\text { randomizado } \\
\text { cruzado }\end{array}$ & $\begin{array}{l}22 \text { adultos con } \\
\text { diagnóstico de EC } \\
\text { (biopsia) en una dieta } \\
\text { con gluten }\end{array}$ & $\begin{array}{l}1,2 \times 10^{10} \text { UFC de Bifibacterium } \\
\text { infantis NLS super strain diariamente } \\
\text { por } 3 \text { semanas vs placebo (capsulas) }\end{array}$ & $\begin{array}{l}\text { 1. Mejoría en síntomas Gl (GSRS) } \\
\text { 2. Cambios en la permeabilidad } \\
\text { intestinal (lactulosa/manitol) } \\
\text { 3. Cambios en IgA-tTG e IgA-DGP } \\
\text { 4. Número de efectos adversos }\end{array}$ \\
\hline $\begin{array}{l}\text { Smecuol y } \\
\text { col. }^{24}\end{array}$ & $\begin{array}{l}\text { Argentina } \\
\text { Un solo centro } \\
\text { Estudio clínico } \\
\text { randomizado } \\
\text { cruzado }\end{array}$ & $\begin{array}{l}12 \text { adultos con } \\
\text { diagnóstico de EC } \\
\text { (biopsia) en DLG por } \\
21 \text { años con síntomas } \\
\text { persistentes }\end{array}$ & $\begin{array}{l}1.2 \times 10^{10} \text { UFC de Bifibacterium } \\
\text { infantis NLS super strain diariamente } \\
\text { por } 3 \text { semanas vs placebo (capsulas) }\end{array}$ & $\begin{array}{l}\text { 1. Mejoría de síntomas específicos de EC } \\
\text { (CSI) } \\
\text { 2. Número de efectos adversos }\end{array}$ \\
\hline
\end{tabular}

Adaptado de Seiler y col. ${ }^{20}$

EC: enfermedad celiaca; DLG: dieta libre de gluten; UFC: unidades formadoras de colonias; CDV: calidad de vida; Gl: gastrointestinal; GSRS: Gastrointestinal Symptoms Rating Scale; IBS: irritable bowel syndrome; IL: interleukina; NLS: Natren Life Start; QOL: Quality of Life; SSS: Symptom Severity Score; CDQ: Score general; TNF-a: factor de necrosis tumoral alfa; tTG: transglutaminasa; DGP: péptidos deamidados de gliadina. 
En conclusión, el uso de probióticos tendría un efecto beneficioso en la mejoría sintomática en la EC, y su administración demostró ser segura. Sin embargo, el grado de certeza de la evidencia actual es bajo, posiblemente relacionado con el bajo número de pacientes estudiados y la heterogeneidad en el diseño de las investigaciones. Por lo tanto, se necesitan estudios clínicos randomizados y controlados, con una muestra poblacional apropiada, y con un diseño riguroso que permitan evaluar el efecto de los probióticos sobre diferentes variables, incluyendo la mejoría de los síntomas intestinales y extraintestinales, la reducción de actividad de la EC, así como también el efecto en la mejoría de la calidad de vida de estos pacientes.

Sostén financiero. MIPS obtuvo una beca interna de Investigacion del Instituto Farncombe y de la division de Gastroenterologia.

Propiedad intelectual. El autor declara que los datos, las tablas y las figuras presentes en el manuscrito son veraces. Asimismo, solicitó autorización y dio reconocimiento al autor de la tabla publicada. ${ }^{20}$

\section{Aviso de derechos de autor}

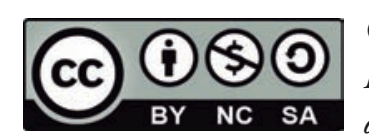

(C) 2021 Acta Gastroenterológica Latinoamericana. Este es un artículo de acceso abierto publicado bajo los términos de la Licencia Creative Commons Attribution (CC BY-NC-SA 4.0), la cual permite el uso, la distribución $y$ la reproducción de forma no comercial, siempre que se cite al autor y la fuente original.

Cite este artículo como: Rueda GH, Pinto-Sánchez MI. Probióticos en enfermedad celiaca: ¿estamos listos para su aplicación en la práctica clínica? Acta Gastroenterol Latinoam. 2021;51(4):394-2. https://doi.org/10.52787/ GQME9827

\section{Referencias}

1. Hill C, Guarner F, Reid G, et al. Expert consensus document. The International Scientific Association for Probiotics and Prebiotics consensus statement on the scope and appropriate use of the term probiotic. Nat Rev Gastroenterol Hepatol. 2014;11(8):506514. doi:10.1038/nrgastro.2014.66

2. Quigley EMM. Prebiotics and Probiotics in Digestive Health. Clin Gastroenterol Hepatol. 2019;17(2):333-344. doi:10.1016/j. cgh.2018.09.028
3. Caminero A, Pinto-Sanchez MI. Host immune interactions in chronic inflammatory gastrointestinal conditions. Curr Opin Gastroenterol. 2020;36(6):479-84. doi:10.1097/MOG.00000 00000000673

4. Ludvigsson JF, Leffler DA, Bai JC, et al. The Oslo definitions for coeliac disease and related terms. Gut. 2013;62(1):43-52. doi:10.1136/gutjnl-2011-301346

5. Singh P, Arora A, Strand TA, et al. Global Prevalence of Celiac Disease: Systematic Review and Meta-analysis. Clin Gastroenterol Hepatol. 2018;16(6):823-36.e2. doi:10.1016/j.cgh. 2017.06.037

6. Parra-Medina R, Molano-Gonzalez N, Rojas-Villarraga $\mathrm{A}$, et al. Prevalence of celiac disease in latin america: a systematic review and meta-regression. PLoS One. 2015;10(5):e0124040. Published 2015 May 5. doi:10.1371/ journal.pone. 0124040

7. Lebwohl B, Sanders DS, Green PHR. Coeliac disease. Lancet. 2018;391(10115):70-81. doi:10.1016/S0140-6736(17) 31796-8

8. Mårild K, Söderling J, Bozorg SR, et al. Costs and Use of Health Care in Patients With Celiac Disease: A Population-Based Longitudinal Study. Am J Gastroenterol. 2020;115(8):125363. doi:10.14309/ajg.0000000000000652

9. Rubio-Tapia A, Hill ID, Kelly CP, Calderwood AH, Murray JA; American College of Gastroenterology. ACG clinical guidelines: diagnosis and management of celiac disease. Am J Gastroenterol. 2013;108(5):656-77. doi:10.1038/ajg.2013.79

10. Nachman F, Mauriño E, Vázquez H, et al. Quality of life in celiac disease patients: prospective analysis on the importance of clinical severity at diagnosis and the impact of treatment. Dig Liver Dis. 2009;41(1):15-25. doi:10.1016/j. dld.2008.05.011

11. Roos S, Liedberg GM, Hellström I, Wilhelmsson S. Persistent Symptoms in People With Celiac Disease Despite Gluten-Free Diet: A Concern?. Gastroenterol Nurs. 2019;42(6):496-503. doi:10.1097/SGA.0000000000000377

12. Leffler DA, Dennis M, Hyett B, Kelly E, Schuppan D, Kelly CP. Etiologies and predictors of diagnosis in nonresponsive celiac disease. Clin Gastroenterol Hepatol. 2007;5(4):445-50. doi:10.1016/j.cgh.2006.12.006

13. Kivelä L, Caminero A, Leffler DA, Pinto-Sanchez MI, TyeDin JA, Lindfors K. Current and emerging therapies for coeliac disease. Nat Rev Gastroenterol Hepatol. 2021;18(3):181-95. doi:10.1038/s41575-020-00378-1

14. Rooks MG, Garrett WS. Gut microbiota, metabolites and host immunity. Nat Rev Immunol. 2016;16(6):341-52. doi:10.1038/ nri.2016.42

15. Caminero A, Verdu EF. Celiac disease: should we care about microbes?. Am J Physiol Gastrointest Liver Physiol. 2019;317(2):G161-70. doi:10.1152/ajpgi.00099.2019

16. W Wacklin P, Laurikka P, Lindfors K, et al. Altered duodenal microbiota composition in celiac disease patients suffering from persistent symptoms on a long-term gluten-free diet. Am J Gastroenterol. 2014;109(12):1933-41. doi:10.1038/ ajg. 2014.355

17. K Klemenak M, Dolinšek J, Langerholc T, et al. Administration of Bifidobacterium breve Decreases the Production of TNF- $\alpha$ in Children with Celiac Disease. Dig Dis Sci. 2015;60(11):338692. doi:10.1007/s10620-015-3769-7 
18. McCarville JL, Dong J, Caminero A, et al. A Commensal Bifidobacterium longum Strain Prevents Gluten-Related Immunopathology in Mice through Expression of a Serine Protease Inhibitor. Appl Environ Microbiol. 2017;83(19):e01323-17. doi:10.1128/AEM.01323-17

19. Klarin B,WulltM, Palmquist I,Molin G, Larsson A, Jeppsson B. Lactobacillus plantarum 299v reduces colonisation of Clostridium difficile in critically ill patients treated with antibiotics. Acta Anaesthesiol Scand. (2008) 52:1096-102. doi: 10.1111/j.13996576.2008.01748

20. Seiler CL, Kiflen M, Stefanolo JP, et al. Probiotics for Celiac Disease: A Systematic Review and Meta-Analysis of Randomized Controlled Trials. Am J Gastroenterol. 2020;115(10):158495. doi:10.14309/ajg.0000000000000749

21. Francavilla R, Piccolo M, Francavilla A, et al. Clinical and microbiological effect of a multispecies probiotic supplementation in celiac patients with persistent IBS-type symptoms: A randomized, double-blind, placebo-controlled, multicenter trial. J Clin Gastroenterol 2019;53(3):e117-25. doi: 10.1097/ MCG.0000000000001023.

22. Harnett J, Myers SP, Rolfe M. Probiotics and the microbiome in celiac disease: A randomised controlled trial. Evid Based Complement Altern Med 2016;2016:9048574. doi:10.1155/ 2016/9048574

23. Smecuol E, Hwang HJ, Sugai E, et al. Exploratory, randomized, double-blind, placebo-controlled study on the effects of Bifidobacterium infantis natren life start strain super strain in active celiac disease. J Clin Gastroenterol. 2013;47(2):139-47. doi:10.1097/MCG.0b013e31827759ac

24. Smecuol E, Constante M, Temprano MP, et al. Effect of Bifidobacterium infantis NLS super strain in symptomatic coeliac disease patients on long-term gluten-free diet - an exploratory study. Benef Microbes. 2020;11(6):527-34. doi:10.3920/ BM2020.0016

25. Palma GD, Capilla A, Nova E, et al. Influence of milk-feeding type and genetic risk of developing coeliac disease on intestinal microbiota of infants: the PROFICEL study. PLoS One. 2012;7(2):e30791. doi:10.1371/journal.pone.0030791

26. Crespo-Escobar P, Mearin ML, Hervás D, et al. The role of gluten consumption at an early age in celiac disease development: a further analysis of the prospective PreventCD cohort study. Am J Clin Nutr. 2017;105(4):890-96. doi:10.3945/ ajcn.116.144352

27. Dydensborg Sander S, Nybo Andersen AM, Murray JA, Karlstad Ø, Husby S, Størdal K. Association Between Antibiotics in the First Year of Life and Celiac Disease. Gastroenterology. 2019;156(8):2217-29. doi:10.1053/j.gastro.2019.02.039

28. Bouziat R, Hinterleitner R, Brown JJ, et al. Reovirus infection triggers inflammatory responses to dietary antigens and development of celiac disease. Science. 2017;356(6333):44-50. doi:10.1126/science.aah5298

29. Gómez-Rial J, Rivero-Calle I, Salas A, Martinón-Torres F. Rotavirus and autoimmunity. J Infect. 2020;81(2):183-89. doi:10.1016/j.jinf.2020.04.041

30. Olivares M, Neef A, Castillejo G, et al. The HLA-DQ2 genotype selects for early intestinal microbiota composition in infants at high risk of developing coeliac disease. Gut. 2015;64(3):406-17. doi:10.1136/gutjnl-2014-306931
31. Di Cagno R, Rizzello CG, Gagliardi F, et al. Different fecal microbiotas and volatile organic compounds in treated and untreated children with celiac disease. Appl Environ Microbiol. 2009;75(12):3963-71. doi:10.1128/AEM.02793-08

32. Wacklin P, Kaukinen K, Tuovinen E, et al. The duodenal microbiota composition of adult celiac disease patients is associated with the clinical manifestation of the disease. Inflamm Bowel Dis. 2013;19(5):934-41. doi:10.1097/MIB .0b013e31828029a9

33. Quagliariello A, Aloisio I, Bozzi Cionci N, et al. Effect of Bifidobacterium breve on the Intestinal Microbiota of Coeliac Children on a Gluten Free Diet: A Pilot Study. Nutrients. 2016;8(10):660. Published 2016 Oct 22. doi:10.3390/nu8100660

34. Olivares M, Castillejo G, Varea V, Sanz Y. Double-blind, randomised, placebo-controlled intervention trial to evaluate the effects of Bifidobacterium longum CECT 7347 in children with newly diagnosed coeliac disease. Br J Nutr. 2014;112(1):30-40. doi:10.1017/S0007114514000609

35. D'Arienzo R, Stefanile R, Maurano F, et al. Immunomodulatory effects of Lactobacillus casei administration in a mouse model of gliadin-sensitive enteropathy. Scand J Immunol. 2011;74(4):33541. doi:10.1111/j.1365-3083.2011.02582.x

36. Francavilla R, De Angelis M, Rizzello CG, Cavallo N, Dal Bello F, Gobbetti M. Selected probiotic lactobacilli have the capacity to hydrolyze gluten peptides during simulated gastrointestinal digestion. Appl Environ Microbiol. (2017) 83:e00376-17. doi: 10.1128/AEM.00376-17

37. Caminero A, Galipeau HJ, McCarville JL, et al. Duodenal Bacteria From Patients With Celiac Disease and Healthy Subjects Distinctly Affect Gluten Breakdown and Immunogenicity. Gastroenterology. 2016;151(4):670-83. doi:10.1053/j.gastro.2016.06.041

38. Laparra JM, Olivares M, Gallina O, Sanz Y. Bifidobacterium longum CECT 7347 modulates immune responses in a gliadin-induced enteropathy animal model. PLoS ONE. 2012; 7:e30744.. doi: 10.1371/journal.pone.0030744

39. De Palma G, Cinova J, Stepankova R, Tuckova L, Sanz Y. Pivotal Advance: Bifidobacteria and Gram-negative bacteria differentially influence immune responses in the proinflammatory milieu of celiac disease. J Leukoc Biol. 2010;87(5):765-778. doi:10.1189/ jlb.0709471

40. Primec M, Klemenak M, Di Gioia D, et al. Clinical intervention using Bifidobacterium strains in celiac disease children reveals novel microbial modulators of TNF- $\alpha$ and short-chain fatty acids. Clin Nutr. 2019;38(3):1373-81. doi:10.1016/j. clnu.2018.06.931

41. Lindfors $\mathrm{K}$, Blomqvist $\mathrm{T}$, Juuti-Uusitalo $\mathrm{K}$, et al. Live probiotic Bifidobacterium lactis bacteria inhibit the toxic effects induced by wheat gliadin in epithelial cell culture. Clin Exp Immunol. 2008;152(3):552-8. doi:10.1111/j.13652249.2008.03635.x

42. Orlando A, Linsalata M, Notarnicola M, Tutino V, Russo F. Lactobacillus GG restoration of the gliadin induced epithelial barrier disruption: the role of cellular polyamines. BMC Microbiol. 2014;14:19. doi:10.1186/1471-2180-14-19

43. Papista C, Gerakopoulos V, Kourelis A, et al. Gluten induces coeliac-like disease in sensitised mice involving IgA, CD71 and transglutaminase 2 interactions that are prevented by probiotics. Lab Invest. 2012;92(4):625-35. doi:10.1038/labinvest.2012.13 
44. Caminero A, McCarville JL, Zevallos VF, et al. Lactobacilli degrade wheat amylase trypsin inhibitors to reduce intestinal dysfunction induced by immunogenic wheat proteins. Gastroenterology. 2019;156(8):2266-80. doi:10.1053/j.gastro.2019.02.028

45. Oscarsson E, Håkansson Å, Andrén Aronsson C, Molin G, Agardh D. Effects of probiotic bacteria Lactobacillaceae on the gut microbiota in children with celiac disease autoimmunity: A placebo-controlled and randomized clinical trial. Front Nutr. 2021;8:680771. doi:10.3389/fnut.2021.680771

46. Herrán AR, Pérez-Andrés J, Caminero A, et al. Gluten-degrading bacteria are present in the human small intestine of healthy volunteers and celiac patients. Res Microbiol. 2017;168(7):673-84. doi:10.1016/j.resmic.2017.04.008
47. Caminero A, Meisel M, Jabri B, Verdu EF. Mechanisms by which gut microorganisms influence food sensitivities. Nat Rev Gastroenterol Hepatol. 2019;16(1):7-18. doi:10.1038/s41575018-0064-z

48. Lamas B, Hernandez-Galan L, Galipeau HJ, et al. Aryl hydrocarbon receptor ligand production by the gut microbiota is decreased in celiac disease leading to intestinal inflammation. Sci Transl Med. 2020;12(566):eaba0624. doi: 10.1126/scitranslmed. aba0624. 